\title{
Ciclos Econômicos e Crimes contra o Patrimônio: uma Análise quase Experimental
}

\section{Business Cycles and Crimes against Property: a Quasi- experimental Approach}

\author{
Cristiano Aguiar de Oliveira ${ }^{a}$ \\ Iuri Capa Verde Costa ${ }^{a}$
}

\begin{abstract}
Resumo: Este artigo busca evidências das relações entre ciclos econômicos e crimes contra o patrimônio a partir de um quase experimento gerado pela implantação do Polo Naval no município brasileiro de Rio Grande (RS) a partir do ano de 2006. Para esse fim, utilizam-se modelos estruturais de séries de tempo estimados através do Filtro de Kalman para decompor e identificar quebras estruturais nas séries de furtos e roubos registrados mensalmente no município, no período compreendido entre janeiro de 2002 e dezembro de 2015. Os resultados indicam a existência de quebras estruturais tanto em furtos, quanto em roubos, que podem estar associadas às mudanças geradas na economia em decorrência da implementação do Polo Naval. O artigo traz evidências de que o início da construção da plataforma P-53 coincide com a redução de forma permanente de 80 furtos mensais e que a construção da plataforma P-55 coincide com a redução permanente de 62 roubos mensais registrados no município. $\mathrm{O}$ artigo conclui que existem evidências de um efeito motivação em que a melhora nas condições econômicas do município foi capaz de gerar efeitos dissuasórios que reduziram os crimes contra o patrimônio.
\end{abstract}

Palavras-chave: Polo Naval. Crime. Quase-experimento. Quebras Estruturais.

\begin{abstract}
This paper seeks evidence of the relationship between economic cycles and property crimes in a quasi-experiment generated by the shipbuilding industry installed in the Brazilian city of Rio Grande (RS) from the year 2006. For this purpose, it uses structural time series models estimated by the Kalman Filter to decompose and to identify structural breaks in the thefts and robberies monthly registered in the municipality between January 2002 and December 2015. The results indicate the existence of structural breaks both in thefts and robberies that may be associated with the changes generated in the economy due to the implementation of the shipbuilding industry. The paper provides evidence that the start of construction of the P-53 platform coincide with 80 monthly thefts while that the construction of the P-55 platform coincide with the reduction in 62 monthly robberies registered in the municipality. The paper concludes that there is evidence of a motivation effect in which the improvement in the economic conditions of the municipality was able to generate deterrence effects that reduced the property crimes.
\end{abstract}

Keywords: Naval pole. Crime. Quasi-experiment. Structural breaks.

a Universidade Federal do Rio Grande (Furg), Instituto de Ciências Econômicas, Administrativas e Contábeis, Programa de Pós-Graduação em Economia Aplicada. Rio Grande, Rio Grande do Sul, Brasil. 


\section{Introdução}

Desde quando Becker (1968), em seu artigo seminal, criou as bases para o surgimento de uma nova linha de pesquisa na ciência econômica, a Economia do Crime trata o crime como uma atividade econômica alternativa ao mercado de trabalho legal. Como tal, envolve uma decisão individual que avalia custos e benefícios em que as suas possibilidades de inserção e ganhos no mercado de trabalho legal seriam vistas pelos agentes como uma forma de custo de oportunidade. Seguindo esse raciocínio, muitos estudos têm buscado encontrar relações entre condições econômicas agregadas caracterizadas por ciclos econômicos e o crime. No fundo, o que se busca são evidências empíricas agregadas com variáveis sugeridas por um modelo individual que avalia a mentalidade e o sistema de incentivos de um potencial criminoso. Embora possa parecer que a agregação de comportamentos individuais seja uma dedução trivial, o fato é que esse mecanismo de agregação é um pouco mais complexo, porque, em princípio, todos os indivíduos são ao mesmo tempo potenciais vítimas e potenciais criminosos. Dessa forma, por exemplo, mudanças em variáveis agregadas, tais como o nível de emprego, podem alterar simultaneamente os custos de oportunidade para alguns indivíduos e os benefícios do crime para outros.

Considerando esses aspectos, estudos que relacionam o crime com as variáveis econômicas de uma região podem gerar resultados diferentes do que se esperaria de um modelo para indivíduos, por exemplo, observando uma relação positiva entre crime e renda. Além desses problemas, estudos empíricos desse tipo ainda têm de lidar com os conhecidos problemas causados pela violação da exogeneidade estrita em modelos econométricos, que podem ter sua origem, por exemplo, na omissão de variáveis relevantes que variam no tempo e que possuam alguma correlação com variáveis independentes incluídas nos modelos. Como o crime é multifatorial, tal problema é recorrente e são poucas as oportunidades em que os pesquisadores conseguem observar uma fonte de variação exógena capaz de identificar relações de causalidade. Todavia, é cada vez mais comum a utilização de quase experimentos para esse propósito, como, por exemplo, nos estudos de Di Tella e Schargrodsky (2004), que utilizam o ataque ocorrido ao centro judaico ocorrido em 1994 para avaliar os impactos da polícia no crime. Por sua vez, mudanças na renda e no nível de emprego gerados por uma fonte de variação exógena na forma de um quase experimento são raras, e, ao que se sabe, não há nenhum estudo que utilize um evento desse tipo para relacionar essas variáveis agregadas com o crime.

Nesse contexto, o município do Rio Grande passou por momentos de grandes transformações econômicas com o crescimento do emprego, da renda e da migração trazido pela implantação do que veio a ser chamado de Polo Naval. O Polo Naval consistia em um conjunto de três estaleiros, Honório Bicalho (HB) e Es- 
taleiros Rio Grande (ERG1 e ERG2), capazes de construir plataformas oceânicas do tipo Floating production, storage and offloading (FPSO) para a extração de petróleo pela Petrobras. Esse processo foi iniciado, em 2006, com a construção do dique seco do estaleiro ERG1, o segundo maior dique seco do mundo, e com o início das operações do estaleiro HB através da construção do casco da plataforma P-53 em 2007.

Os impactos dessa mudança podem ser vistos na Tabela 1. De 2002 a 2013, o PIB do município cresceu cerca de $80 \%$ em termos reais e o número de trabalhadores formais cresceu cerca de $93 \%$. Nesse período é possível identificar pelo menos dois momentos de crescimento abrupto: um que compreende os anos de 2007 e 2008, com o início das obras para a construção do dique seco do estaleiro ERG1 e a construção da plataforma P53 pelo estaleiro HB, e outro momento que compreende os anos de 2012 e 2013, com a construção simultânea dos cascos das plataformas P55, P58, P63 pelo mesmo estaleiro e as plataformas P66 e P67 pelos estaleiros ERG1 e ERG2, respectivamente. O primeiro momento mostra uma taxa de crescimento econômico acumulado de quase $54 \%$ em dois anos e o segundo, um crescimento ecnômico um pouco menor, cerca de $31 \%$ ao longo de dois anos, porém com forte crescimento do emprego formal, cerca de $25 \%$.

Tabela 1 - PIB do município de Rio Grande e sua taxa de crescimento real anual (2002-2014)

\begin{tabular}{ccccc}
\hline Ano & PIB nominal* & $\Delta \%$ PIB real** & $\begin{array}{c}\text { Pessoal } \\
\text { ocupado*** }\end{array}$ & $\begin{array}{c}\Delta \% \text { Pessoal } \\
\text { ocupado }\end{array}$ \\
\hline 2002 & $1.873 .915,74$ & - & 29143 & - \\
2003 & $2.453 .544,58$ & $19,14 \%$ & 30530 & $4,76 \%$ \\
2004 & $2.430 .940,11$ & $-13,09 \%$ & 33015 & $8,14 \%$ \\
2005 & $2.236 .434,31$ & $-14,66 \%$ & 33745 & $2,21 \%$ \\
2006 & $2.513 .033,05$ & $4,53 \%$ & 35093 & $3,99 \%$ \\
2007 & $3.685 .379,56$ & $37,44 \%$ & 36073 & $2,79 \%$ \\
2008 & $4.391 .320,94$ & $11,99 \%$ & 36334 & $0,72 \%$ \\
2009 & $4.592 .347,24$ & $-3,97 \%$ & 37303 & $2,67 \%$ \\
2010 & $5.143 .120,72$ & $4,28 \%$ & 39859 & $6,85 \%$ \\
2011 & $5.417 .421,73$ & $-3,01 \%$ & 44976 & $12,84 \%$ \\
2012 & $6.460 .758,15$ & $10,12 \%$ & 52897 & $17,61 \%$ \\
2013 & $8.147 .934,39$ & $19,09 \%$ & 56354 & $6,54 \%$ \\
2014 & $7.357 .681,05$ & $-15,21 \%$ & 48567 & $-13,82 \%$ \\
\hline
\end{tabular}

Fonte: FEE e RAIS.

Nota: "A preços de mercado; * "calculada a partir do deflator do PIB calculado pelo IBGE;

$* * *$ empregos formais com carteira assinada. 
Essa amostragem de informações permite evidenciar os efeitos da implantação do Polo Naval no município e seus respectivos efeitos multiplicadores na renda e na quantidade de empregos. Como pode ser observado na Tabela 1, houve uma mudança brusca nas variáveis econômicas no período tanto na implantação do Polo Naval, quanto na redução significativa de suas atividades, e, portanto, essas alterações geram a possibilidade de se trabalhar com um quase experimento, uma vez que essas mudanças ocorridas no município se assemelham ao período de ápice de um ciclo econômico, porém são exógenas e apresentam uma variação muito mais abrupta do que as previstas pelos tradicionais modelos de ciclos de negócios. ${ }^{1}$ Quando isso ocorre é possível discriminar o comportamento de série temporal em antes e depois da mudança (intervenção). Esse processo de discriminação, conhecido como interrupted time series analysis, possui um bom potencial de validade interna e é um dos métodos quase experimentais mais úteis para se avaliar mudanças que afetam um local quando não é possível distinguir tratados e não tratados (SHADISH; COOK; CAMPBELL, 2002; CAMPBELL; STANLEY, 2015).

Por sua vez, a discussão a respeito das relações entre ciclos econômicos e criminalidade tem uma ampla literatura internacional, tal como os estudos de Grogger (1998), Funk e Kugler (2000), Raphael e Winter-Ebmer (2001), Levitt (2001), Melick (2003), Edmark (2005), Mocan e Bali (2010), Dongil (2006), Sookram et al. (2009), Dritsakis e Gkanas (2009), Zwienen (2011), entre outros. Diferentemente do cenário internacional, na literatura nacional são poucos os trabalhos que tratam do tema com o uso de séries temporais. Cabe citar os esforços feitos por Pereira e Carreira Fernandez (2000), Araújo Júnior e Shikida (2010), Santos e Kassouf (2011), Johnston e Oliveira (2016) e Arcaro e Oliveira (2016). No entanto, nenhum dos trabalhos citados na literatura nacional estuda a relação entre ciclos econômicos e o crime em uma região que tenha passado pelas mudanças econômicas que o município de Rio Grande experimentou.

Nesse contexto, cabe questionar: será que essas mudanças abruptas nas condições econômicas do município foram capazes de alterar o comportamento de seus agentes a ponto de mudar as suas decisões a respeito de praticar crimes? Diante da possibilidade de tratar com um quase experimento, este trabalho visa contribuir para a literatura ao avaliar os impactos econômicos que ocorreram a partir da implementação do Polo Naval nos crimes contra o patrimônio do município, com dados mensais. Para esse fim, utilizam-se modelos estruturais de séries de tempo estimados com o uso do filtro de Kalman (FK) para se decompor as séries de crime e para se identificar possíveis quebras estruturais. ${ }^{2}$

\footnotetext{
1 Um dos debates mais controversos na Teoria Econômica é o que diz respeito à origem dos ciclos de negócios. Existem muitos modelos teóricos, porém os mais influentes são certamente os modelos novo-keynesianos e novo-clássicos. e Koopman (2012), tem sido aplicado para avaliar os impactos de intervenções em várias áreas
} 
A escolha dessa metodologia se justifica pela falta de informações econômicas com periodicidade mensal e de municípios comparáveis para se construir um painel de dados que permita uma análise de controle sintético ou de diferenças em diferenças. Ademais, trata-se de uma metodologia mais flexível e transparente do que a tradicional metodologia de interrupted time series, que se baseia nos tradicionais modelos Arima de Box e Jenkins (1976), tais como os modelos de Gottman (1981) e Crosbie (1993). Modelos estruturais de séries de tempo permitem trabalhar com séries não estacionárias no seu nível, ou seja, com tendência estocástica, algo que não é possível nos modelos Arima, e permitem lidar com séries com alta variabilidade, além de permitir a modelagem de intervenções através de critérios estatísticos transparentes (HARVEY, 1989; VUIJIC; COMMANDEUR; KOOPMAN, 2016).

O método consiste em decompor uma série de tempo em componentes determinísticos e estocásticos que incluem seu nível, inclinação, tendência, sazonalidade, ciclos, choques e, principalmente, quebras estruturais. Este último componente da estrutura de séries de crimes contra o patrimônio é o de maior interesse neste estudo, pois se objetiva identificar a dinâmica dos impactos de uma mudança abrupta nas condições econômicas do município sobre as suas estatísticas de furtos e roubos. Nesse contexto, a estratégia de identificação adotada neste estudo é realizar intervenções manuais baseadas em datas relevantes (início de operações e chegadas e saídas de plataformas) e intervenções "automáticas" baseadas em critérios estatísticos. A coincidência entre as mudanças econômicas e as mudanças nos registros de crimes é interpretada como um forte indício de causalidade. ${ }^{3}$

O artigo está organizado em mais quatro seções, além desta introdução: a segunda seção aborda os principais aspectos teóricos da teoria econômica do crime, assim como uma breve revisão da literatura empírica que relaciona emprego e renda com crimes em termos agregados; a terceira seção explica a metodologia utilizada, dando ênfase aos modelos estruturais de séries de tempo com quebras estruturais estimados com o uso do FK; a quarta seção apresenta os principais resultados obtidos; por fim, a quinta seção traça as considerações finais a respeito dos impactos da implementação do Polo Naval nos crimes contra o patrimônio no município de Rio Grande.

do conhecimento, dentre as quais cabe citar os estudos de Harvey e Durbin (1986), que analisa os efeitos da lei que tornou obrigatório o uso de cinto de segurança na Grã-Bretanha nas mortes de motoristas, e o estudo de Vujic, Commandeur e Koopman (2016), que analisa os efeitos da revogação da liberdade condicional no estado da Virginia nos crimes registrados no estado.

3 Mais adiante no artigo, a validade interna dos resultados obtidos é discutida em maiores detalhes. 


\section{Revisão de Literatura}

Muitos autores tratam de relacionar a teoria econômica e a criminalidade. $\mathrm{O}$ marco dos estudos relacionando os crimes com a teoria econômica é o trabalho de Becker (1968). Nesse estudo, o autor traz a suposição da racionalidade do indivíduo em sua decisão de cometer ou não um ato ilícito. Becker afirma que um indivíduo só irá cometer um ato ilegal se o benefício esperado por ele for maior que o benefício que terá se empregar o seu tempo em uma atividade legal. Segundo o autor, existe uma função que pode ser considerada como a oferta de crimes, que depende da probabilidade de o indivíduo ser punido ao cometer o ato ilícito, do grau de severidade dessa possível punição e de outras variáveis tais como a própria renda que esse indivíduo tenha à disposição, tanto na atividade legal (custo de oportunidade), quanto na ilegal (benefício do crime).

É possível afirmar que, partir de Becker (1968), houve um impulso dos estudos que objetivavam relacionar crimes com variáveis econômicas. Um dos precursores é o estudo de Ehrlich (1973). Em seu artigo, busca avaliar os efeitos que variáveis relacionadas à renda e à medidas de desigualdade podem causar nas variações das oportunidades da atividade criminosa. Uma das principais contribuições desse estudo é a ideia de que os indivíduos podem gerar uma alocação ótima do tempo para a atividade ilegal, assim como pode ser gerado uma alocação ótima de tempo para a atividade legal. Portanto, é possível também que indivíduos optem por exercer atividades nos dois setores de forma simultânea ou alternada.

Outra proposição de Becker (1968) relevante para o presente estudo é a sua divisão dos crimes em dois grupos: o lucrativo/econômico e o não lucrativo/não econômico. Crimes lucrativos são aqueles que possuem referência com algum valor monetário. Seriam os casos de roubos, furtos, extorsão, estelionato, entre outros crimes. Por sua vez, os crimes não lucrativos seriam aqueles que não trazem relação com algum valor monetário, embora possam trazer benefícios psíquicos, tal como o estupro.

Se o crime lucrativo faz parte da questão econômica, sendo inclusive objeto de estudo da Economia, as questões nucleares que emergem dessa contextualização resumem-se em: quais as circunstâncias socioeconômicas da escolha ocupacional entre o setor legal e o ilegal da economia? E por que os indivíduos decidem praticar crimes lucrativos? A resposta a esses questionamentos passa pelos incentivos recebidos em que forças de dissuasão podem alterar as decisões de engajamento no setor ilegal, dentre as quais a renda e o emprego podem ter um papel relevante.

Segundo Arcaro e Oliveira (2016), a geração de renda e o nível de emprego em uma região influenciam tanto os benefícios do crime quanto os custos de oportunidade e, dessa forma, podem gerar relações com efeitos ambíguos nos crimes 
lucrativos. Os autores destacam a existência de um "efeito oportunidade", que implica uma relação negativa entre crime e desemprego, na qual em um período de aumento do desemprego e redução da renda há uma redução no número de vítimas e nos valores potenciais que podem ser obtidos através de ações criminosas (BRITT, 1994; MELICK, 2003). Existe também um "efeito motivação", em que uma redução nos empregos no setor legal (REILLY; WITT, 1992; PAPPS; WINKELMANN, 2000; RAPHAEL; WINTER-EBMER, 2001; EDMARK, 2005) e na renda (GROGGER, 1998; MACHIN; MEGHIR, 2004; GOULD; WEINBERG; MUSTARD, 2002) reduzem os custos de oportunidade de potenciais criminosos, aumentando as chances de se optar por uma atividade ilegal.

Baseando-se nesses princípios, vários estudos tentam avaliar as relações entre os crimes contra o patrimônio (lucrativos) com ciclos e flutuações econômicas, dentre os quais cabe destacar os trabalhos de Raphael e Winter-Ebmer (2001), Narayan e Smyth (2004), Mocan e Bali (2010), Dongil (2006) e Zwienen (2011). Raphael e Winter-Ebmer (2001) fazem um estudo sobre o efeito do desemprego sobre as taxas de sete crimes nos estados americanos. Como principal resultado do estudo apresenta a presença de efeitos positivos do desemprego sobre os índices de criminalidade contra o patrimônio, que são estáveis em todas as especificações do modelo. Uma das principais conclusões do estudo é que o declínio nas taxas de criminalidade contra o patrimônio, durante a década de 1990, pode ser atribuído ao declínio da taxa de desemprego.

Narayan e Smyth (2004) aplicam o teste de causalidade de Granger para analisar diferentes categorias de crime e relacioná-las com as taxas de desemprego juvenil e o nível de ganho masculino semanal na Austrália. Como resultado, constata que crimes como roubos de veículo, fraudes e homicídios são cointegrados com os índices de desemprego juvenil masculino e com o salário médio semanal do sexo masculino, portanto há uma relação de longo prazo entre essas variáveis.

Mocan e Bali (2010) fazem um estudo buscando identificar a presença de ciclos de criminalidade assimétricos com séries temporais de dados agregados dos Estados Unidos e também com dados da cidade de Nova Iorque. Os autores mostram que há um efeito motivação, mas que os crimes contra a propriedade possuem uma relação assimétrica, em que aumentos nesses índices são mais abruptos com a melhora nas condições econômicas e suas diminuições ocorrem de forma lenta e gradual quando há uma piora nas condições econômicas. Os autores mostram que as variáveis desemprego, PIB real per capita e força policial são as que possuem maior influência na criminalidade no período estudado.

Dongil (2006) faz um estudo buscando analisar os efeitos que as condições econômicas podem ter nos índices de criminalidade na Coreia do Sul a partir de dados trimestrais com o teste de causalidade de Granger. Os resultados sugerem que a desigualdade de renda e o desemprego têm efeito positivo nos roubos, en- 
quanto o nível de renda tem um resultado contrário, ou seja, encontra um efeito motivação para roubos.

Zwienen (2011), assim como Mocan e Bali (2010), busca identificar ciclos assimétricos na relação entre índices de desemprego e crimes. Os resultados obtidos pelo estudo são interessantes porque mostram que há uma assimetria reversa nos ciclos de crimes para homens, com idade abaixo de 25 anos e que praticam os crimes de arrombamento e invasão. O autor identifica que ao longo de períodos de recuperação econômica há um forte efeito oportunidade em que as taxas de criminalidade crescem mais do crescem em períodos de recessão, através de um efeito motivação.

Na literatura nacional, Pereira e Carreira Fernandez (2000) fazem uso de modelos multivariados de séries de tempo para estudar crimes registrados na Grande São Paulo. Os resultados mostram a existência de efeitos motivação, em que o aumento dos índices de desemprego e a redução do rendimento médio do trabalho contribuíram para o crescimento do crime na região, tanto para os modelos que utilizaram crimes agregados quanto para os modelos que utilizaram crimes específicos, tais como o furto e o roubo de veículos.

Apesar de não trabalhar diretamente com metodologias de séries de tempo, Araújo Júnior e Shikida (2010) seguem a mesma linha de Mocan e Bali (2010) e buscam identificar ciclos assimétricos nos municípios de Minas Gerais através de um modelo em painel dinâmico. Os autores encontram evidências de efeitos motivação e de assimetria nos impactos do ciclo econômico em que aumentos no desemprego geram um aumento em crimes contra o patrimônio menor que as quedas nos mesmos quando há uma redução do desemprego. Ou seja, os resultados permitem inferir que potenciais criminosos envolvidos em crimes contra o patrimônio têm maior facilidade em voltar ao mercado de trabalho legal nos períodos de crescimento econômico do que retornar ao crime em um período recessivo.

Santos e Kassouf (2011), através de um modelo autorregressivo vetorial com correção de erro, analisam o comportamento do crime em São Paulo. Os autores concluem que no longo prazo há um efeito motivação em que o crime possui uma relação positiva com a taxa de desemprego e uma relação negativa com variáveis de dissuasão (probabilidade e severidade da pena).

Em estudos para o Rio Grande do Sul, Johnston e Oliveira (2016) estudam os impactos do aprisionamento pela polícia nos roubos registrados na região metropolitana de Porto Alegre (RMPA). No entanto, os autores também avaliam os impactos das variáveis renda e desemprego nos roubos e não encontram uma relação significativa para o desemprego, embora encontrem uma relação positiva entre renda e roubos, ou seja, identificam um efeito oportunidade. Por sua vez, Arcaro e Oliveira (2016) buscam analisar a relação entre os efeitos dissuasórios do 
mercado de trabalho, como renda real e taxa de desemprego com as quedas nos totais de furtos e roubos na última década na RMPA. Como resultado encontrou uma relação de longo prazo entre as variáveis econômicas e de crime e, como principal contribuição, mostra que, assim como Johnston e Oliveira (2016), variações na renda impactam os roubos através do efeito oportunidade e variações na taxa de desemprego afetam o número de furtos através do efeito motivação na RMPA.

Portanto, considerando-se a literatura empírica existente, é possível concluir que efeitos motivação são dominantes, embora existam alguns estudos para a RMPA que indicam um possivel efeito oportunidade. Todavia, cabe salientar que, com exceção dos estudos que utilizam dados em painel, os demais sofrem com potenciais problemas de endogeneidade causados pela omissão de variáveis relevantes. Portanto, o presente estudo busca contribuir para a literatura ao trazer uma evidência utilizando uma análise quase experimental que permite minimizar potenciais problemas causados pela violação da condição de exogeneidade estrita de modelos lineares de regressão. A próxima seção descreve com maiores detalhes a metodologia proposta no presente estudo e como pretende resolver esses e outros problemas comuns que surgem nas tentativas de se realizar inferências causais entre ciclos econômicos e crimes lucrativos.

\section{Metodologia}

Este estudo utiliza o modelo econométrico conhecido como modelo estrutural de séries temporais. Seguindo-se esse modelo, cada tipo de crime é uma série temporal univariada que pode ser representada na seguinte equação de medida:

$$
\mathrm{y}_{\mathrm{t}}=\mu_{\mathrm{t}}+\psi_{\mathrm{t}}+\gamma_{\mathrm{t}}+\varepsilon_{\mathrm{t}}
$$

em que $\mu_{\mathrm{t}}$ é a tendência, $\psi_{\mathrm{t}}$ é o ciclo, $\gamma_{\mathrm{t}}$ é o componente sazonal e $\varepsilon_{\mathrm{t}}$ é o erro que reflete os choques sofridos pela série. Segundo Jalles (2009), uma vantagem da modelagem de séries temporais com o uso de modelos estruturais é a sua flexibilidade em identificar diferenças no comportamento de uma série considerando os seus diferentes componentes como processos estocásticos governados por perturbações aleatórias, de forma que cada componente tem uma equação de transição, como segue:

Tendência $\left(\mu_{\mathrm{t}}\right)$ : esse componente se refere a parte capaz de captar movimentos suaves, em longos períodos de tempo dentro da série temporal (MAIA, 2003). Um modelo de tendência estocástica pode ser escrito por: 


$$
\begin{aligned}
& \mu_{t}=\mu_{t-1}+\beta_{t}+\eta_{t} \\
& \beta_{t}=\beta_{t-1}+\varpi_{t}
\end{aligned}
$$

no qual $\mu_{\mathrm{t}}$ define o nível da tendência e $\beta_{\mathrm{t}}$ define a sua inclinação. Por sua vez, $\eta_{t}$ é um componente estocástico da equação de transição do nível e $\varpi_{t}$ é o componente estocástico da equação de transição da inclinação;

b) ciclo $\left(\psi_{\mathrm{t}}\right)$ : em muitas séries de tempo econômico, é importante fazer a distinção entre uma tendência de longo prazo e os movimentos cíclicos ou de curto prazo, que podem ter uma especificação determinística ou estocástica. Um ciclo determinístico descreve perfeitamente um comportamento periódico com uma dada frequência, de forma que pode ser descrito como uma função de senos e cossenos do seguinte tipo:

$$
\psi_{t}=\alpha \cos (\lambda t)+\beta \operatorname{sen}(\lambda t)
$$

a partir dessa equação, é possível adicionar componentes estocásticos, assim como um fator de amortecimento dentro da equação, tornando o ciclo mais flexível e capaz de se adaptar a várias formas funcionais. Dessa maneira, tem-se:

$$
\left(\frac{\psi t}{\psi t *}\right)=\rho\left(\frac{\cos \lambda}{-\sin \lambda} \frac{\sin \lambda}{\cos \lambda}\right)\left(\frac{\psi t-1}{\psi * t-1}\right)+\left(\frac{\kappa t}{\kappa t *}\right)
$$

em que $\rho$ é o parâmetro que determina o decaimento (amortecimento) do ciclo, $\lambda$ é o parâmetro que determina a frequência do ciclo e $\kappa_{t}$ é o componente estocástico erro da equação de transição do ciclo;

c) sazonalidade $\left(\gamma_{t}\right)$ : trata-se de um movimento que apresenta um padrão repetido ao longo de subperíodos de um período de tempo considerado. Seguindo-se Koopman et al. (2007), a função para esse componente tem a seguinte forma:

$$
\gamma_{t}=\sum_{j=1}^{s-1} \gamma_{t-j}+\delta_{t}
$$

na qual $\delta_{t}$ é o componente estocástico da equação de transição da sazonalidade;

d) erro $(\varepsilon t)$ : o erro, ou também chamado de movimento aleatório dentro de uma série, é definido por Maia (2003) como um deslocamento esporádico da série temporal, que não é captado por nenhum dos outros três componentes: tendência, ciclo e sazonalidade. Os erros são justamente os componentes que se tenta minimizar dentro de qualquer método de estimação. 
Logo, seguindo-se essas especificações, qualquer série de tempo poderia ser descrita na forma de espaço-estado, ou seja, com uma equação de medida e equações de transição para cada componente. Especificamente no modelo a ser usado neste estudo, além dos componentes estruturais serão acrescentadas intervenções de forma que seja possível captar possíveis quebras estruturais causadas pela implementação do Polo Naval nos níveis de criminalidade. Assim, a série de tempo dos crimes registrados no município pode ser decomposta da seguinte forma:

$$
\mathrm{y}_{\mathrm{t}}=\mu_{\mathrm{t}}+\psi_{\mathrm{t}}+\gamma_{\mathrm{t}}+\text { Intervenções }+\varepsilon_{\mathrm{t}}
$$

Esse modelo possui um conjunto de variáveis latentes que pode, por exemplo, ser estimado utilizando-se o FK. O FK é um algoritmo recursivo capaz de alcançar a solução para problemas lineares que são relacionados à filtragem de dados discretos com aplicações em várias situações. Segundo Morettin e Toloi (1981), a utilização do FK pode ser feita em problemas de previsão em que a série temporal é modelada por uma média, que varia no tempo e está superposta a um ruído aditivo. A média é obtida, por hipótese, através de combinações lineares de funções conhecidas cujos parâmetros são desconhecidos. Dessa maneira, o FK pode ser descrito como um conjunto de equações matemáticas constituinte de um processo recursivo eficiente de estimação que minimiza o quadrado dos erros. ${ }^{4}$ Para ser aplicado, é necessário que os modelos estejam escritos sob a forma de espaço-estado tal como descrito na equação 6, ou em sua forma matricial, dada por:

$$
\mathrm{Y}_{\mathrm{t}}=\mathrm{Z}_{\mathrm{t}} \mathrm{X}_{\mathrm{t}}+\mathrm{d}_{\mathrm{t}}+\varepsilon_{\mathrm{t}} \text { com } t=1,2, \ldots, \mathrm{T}
$$

na qual $Z_{\mathrm{t}}$ é uma matriz $N \times m$, $\mathrm{d}_{\mathrm{t}}$ é um vetor $N \times 1, \varepsilon_{\mathrm{t}}$ é um vetor serialmente não correlacionado com média zero e matriz de covariância $\mathrm{H}_{\mathrm{t}}$ e $\mathrm{X}_{\mathrm{t}}$ é um vetor $m \times$ 1 que contém as variáveis de estado não observáveis. Se as variáveis de estado (componentes estruturais) podem ser representadas através de um processo markoviano de ordem um, tal como as equações de transição descritas pelas equações 2, 3, 4, e 5, dessa maneira a versão matricial que representa esse conjunto de equações de transição é dada por:

$$
\mathrm{X}_{\mathrm{t}}=\mathrm{T}_{\mathrm{t}} \mathrm{X}_{\mathrm{t}-1}+\mathrm{C}_{\mathrm{t}}+\mathrm{R}_{\mathrm{t}} \mathrm{N}_{\mathrm{t}} \quad \text { com } t=1,2, \ldots, T
$$

na qual $\mathrm{T}_{\mathrm{t}}$ é uma matriz $m \times m, \mathrm{C}_{\mathrm{t}}$ é um vetor $m \times 1, \mathrm{R}_{\mathrm{t}}$ é uma matriz $m \times \mathrm{g}$ e $\mathrm{N}_{\mathrm{t}}$ é um vetor $\mathrm{g} \times 1$ serialmente não correlacionado com média zero e matriz de co-

$4 \quad$ Outro ponto a ser ressaltado é que o FK permite que se possa estimar os parâmetros desconhecidos do modelo através da maximização da verossimilhança via decomposição dos erros de previsão. 
variância $Q_{t}$. Escritos dessa forma, os componentes não observados dos modelos estruturais de séries de tempo podem ser obtidos de forma recursiva pelo FK.

Um processo semelhante é utilizado para identificar quebras estruturais. Quebras estruturais podem ser identificadas visualmente ou a partir do conhecimento de períodos em que mudanças possam ter gerado uma mudança significativa na série de tempo (CAMPBELL; ROSS, 1968). Nesse caso, assume-se que qualquer mudança em outra variável explicativa move-se de forma mais lenta e gradual do que a variável que gerou a intervenção (quebra estrutural). Assim, fazem-se intervenções manuais. Todavia, sempre pode-se incorrer em alguma arbitrariedade ao se escolher tanto a forma de intervenção (nível, tendência ou informação discrepante) quanto as datas. Então, uma alternativa é calcular os erros de previsão e estabelecer um limite crítico para identificar o período como tendo uma informação discrepante. Se os erros de previsão continuam crescendo na medida em que novas previsões são realizadas, então esse é um forte indício de uma quebra estrutural na série de tempo. Assim, uma vez identificada a presença de uma quebra estrutural, basta testar possíveis especificações para a mesma. A especificação que for capaz de minimizar o quadrado dos erros do modelo é a especificação mais indicada.

O software estatístico Structural time series modeller and predictor (Stamp) estima os parâmetros de modelos estruturais com o uso do FK e identifica informações discrepantes e/ou quebras estruturais através do teste de Cusum, que analisa a soma acumulada dos resíduos recursivos. Sempre que essa soma ultrapassa o limite estatístico em determinado período, isso é identificado como uma informação discrepante (outlier) ou uma quebra estrutural. Assim, diferentes especificações são testadas de forma a minimizar o quadrado dos erros e, consequentemente, maximizar o ajustamento do modelo no formato de espaço de estado aos dados observados.

Para que essa identificação de quebra estrutural tenha validade interna, em outras palavras, para que o modelo seja capaz de estimar efeitos de causalidade, é necessário que a mudança gere efeitos permanentes de forma que fiquem evidentem os diferentes comportamentos da série antes e depois da intervenção (LINDEN; ARBOR, 2015). Além disso, a obtenção de validade interna deve superar alguns problemas elencados por Cook e Campbell (1976). Segundo os autores, para que uma interrupção em uma série de tempo (quebra estrutural) possa ser utilizada para inferir a respeito de causalidade, é necessário que o estudo controle mudanças que ocorreram de forma simultânea à intervenção. Por exemplo, muitos estudos que avaliam o impacto de leis ignoram o fato de que a publicidade da lei pode ser mais importante do que ela mesma. Assim, na medida em que a lei perde espaço na mídia, seu efeito dissuasório também perde efeito. Ademais, os autores chamam atenção para o fato de que uma série estacionária e/ou uma série que 
possui muita variabilidade dificultam a identificação de quebras estruturais, uma vez que séries estacionárias revertem para a sua média e uma alta variabilidade pode resultar na identificação de uma quebra estrutural onde na verdade não há.

Todavia, modelos estruturais de séries de tempo são úteis justamente para minimizar esses problemas, pois permitem a identificação robusta de quebras estruturais, uma vez que, diferentemente dos métodos tradicionais que utilizam tendências determinísticas para construir a previsão que serve como série "placebo", ou seja, que permite fazer uma análise contrafactual e mensurar os efeitos do tratamento, os modelos estruturais de séries de tempo tratam todos os componentes como estocásticos e independentes dos erros do modelo. Assim, tem-se uma previsão muito mais precisa que permite uma análise contrafactual robusta baseada na identificação das quebras estruturais apresentadas pelas séries.

Considerando-se esses aspectos, a estratégia de identificação dos impactos da renda e do emprego gerados pela implantação do Polo Naval nos crimes contra o patrimônio no município de Rio Grande utilizada pelo presente estudo consiste em duas etapas, sugeridas por Vujic, Commandeur e Koopman (2016). A primeira estabelece intervenções manuais nas datas relevantes relacionadas aos acontecimentos históricos. A coincidência implica em um forte indício de relação causal entre as mudanças ocorridas na economia do município e suas estatísticas de crimes, pois o método é capaz de isolar os choques que causam a alta variabilidade tanto com séries estacionárias quanto com não estacionárias. Nesse caso, a validade interna só não é completa se houver a omissão de alguma variável relevante que se mova na mesma velocidade da intervenção. Como existe a possibilidade de a intervenção ter efeitos retardados nas séries de interesse, a segunda etapa do estudo utiliza intervenções "automáticas" baseadas em critérios estatísticos. Novamente a coincidência dos períodos de intervenção com os acontecimentos implica um forte indício de relação de causalidade.

\section{Resultados}

Os dados para a execução deste estudo foram extraídos do banco de dados criminalísticos da Secretaria de Segurança Pública do Rio Grande do Sul. São utilizadas as estatísticas mensais agregadas de furtos e roubos registrados no município de Rio Grande no período compreendido entre janeiro de 2002 e dezembro de 2015. 
Figura 1 - Roubos e furtos registrados no município de Rio Grande (2002-2015)


Fonte: SJS/RS.

A Figura 1 mostra a trajetória das séries utilizadas. No período estudado houve um registro médio mensal de 118 e 338 roubos e furtos, respectivamente. Apesar de ser possível identificar algumas mudanças abruptas visualmente, a forma correta de se fazer isso é deixar que os dados indiquem qual é o comportamento, uma vez que as séries apresentam uma grande variabilidade e nem sempre é fácil distinguir mudanças permanentes no nível da série de seus choques ou de seus componentes sazonais.

Conforme já foi comentado, a estratégia de identificação passa por incluir nos modelos estruturais intervenções manuais e automáticas. Assim, inicialmente são apresentados os resultados dos modelos em que houve a inserção de intervenções manuais, ou seja, as intervenções foram imputadas em datas escolhidas conforme os acontecimentos históricos. A partir de pesquisas feitas, foram escolhidas 14 datas, que são mostradas no Quadro 1. Essas datas se referem ao início e ao final das construções mais relevantes do Polo Naval. 
Quadro 1 - Principais construções do Polo Naval no município de Rio Grande

\begin{tabular}{|c|c|c|c|c|c|}
\hline Construções & Empresa & Estaleiro & $\begin{array}{c}\text { Valor } \\
\text { US\$ bilhões }\end{array}$ & Início & Término \\
\hline Dique seco & WTorre & - & 1,2 & Ago./06 & Out./10 \\
\hline P53 & QUIP & HB & 0,8 & Set./07 & Out./08 \\
\hline P55 & QUIP & HB & 1,6 & Set./08 & Out./13 \\
\hline P58 & QUIP & HB & 1,3 & Out./11 & Dez./13 \\
\hline P63 & QUIP & HB & 1,3 & Fev./13 & Jun./13 \\
\hline P66 & ECOVIX & ERG1 & 0,4 & Maio/13 & Dez./14 \\
\hline P67 & ECOVIX & ERG2 & 0,4 & Abr./14 & Set./15 \\
\hline
\end{tabular}

Fonte: Elaboração própria a partir de informações divulgadas na imprensa.

Uma vez estabelecidas as datas das possíveis informações discrepantes e/ou das quebras estruturais, são estimados modelos estruturais de séries de tempo com três tipos de especificações possíveis para essas datas: informações discrepantes (outlier), mudança de inclinação (slope) e mudanças de intercepto (level break). Partindo-se do modelo mais completo para o mais simples, são escolhidos os modelos que possuem especificações com coeficientes estatisticamente significativos. Seguindo-se esse procedimento, se observou que a maior parte das datas não representou mudanças significativas nos dois tipos de crime estudados. No entanto, algumas dessas datas mostraram coeficientes significativos de forma persistente em vários dos modelos que foram estimados. Elas podem ser vistas nos modelos com o melhor ajustamento, apresentados na Tabela 2. 
Tabela 2 - Resultados das estimações com intervenções manuais



Fonte: Elaboração própria.

Os modelos com melhor ajustamento não apresentam o problema de autocorrelação nos resíduos especificados a partir do teste de Ljung-Box, contudo o teste de Jarque-Bera indica que o modelo para roubos não possui normalidade na distribuição dos seus resíduos. Dessa maneira, os resultados desse modelo devem ser interpretados com cautela. ${ }^{5}$ Por sua vez, o modelo para o crime de furto não apresenta problemas de autocorrelação e normalidade dos resíduos, assim trata-se de um modelo capaz de fornecer uma análise robusta.

O modelo 1, para roubos, indica a existência de duas informações discrepantes que coincidem com a chegada da P55 e a saída da P53. Nesses meses, ocorreram cerca de 51 e 85 roubos acima do esperado, respectivamente. Entretanto, o modelo identifica a coincidência de duas quebras estruturais na série de

$5 \quad$ Vale ressaltar que mesmo que a hipótese de normalidade dos resíduos não seja satisfeita, o estimador obtido pelo FK é um estimador linear ótimo que minimiza o erro quadrático médio (HARVEY, 1989). 
roubos nos meses das saídas da P66 e P67. Houve um aumento permanente da ordem de 52 e 48 roubos, respectivamente. Essas coincidências podem ser melhor observadas na Figura 2. Logo, apesar das ressalvas que devem ser feitas pelo comportamento anormal dos resíduos do modelo, há um indício de existência de efeito oportunidade, em que a redução do emprego e da renda implicou um aumento da criminalidade devido à redução dos custos de oportunidade.

Figura 2 - Componentes da série de roubos no município de Rio Grande (modelo 1)
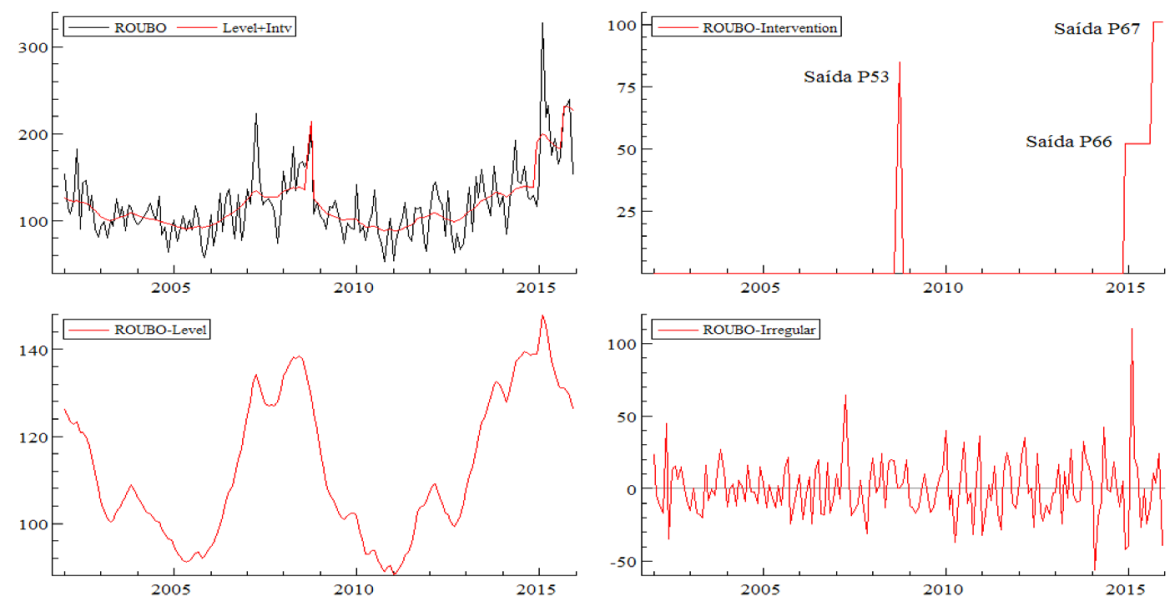

Fonte: Elaboração própria a partir do software Stamp.

Por sua vez, o modelo 2, para furtos, não apresenta qualquer problema relacionado à normalidade dos resíduos e/ou sua autocorrelação. Ele mostra três períodos discrepantes, sendo dois negativos e um positivo. São identificados um aumento de cerca de 63 furtos registrados no mês em que as obras do dique seco foram finalizadas e reduções de cerca de 72 e 84 furtos nos meses em que a P66 e P67 deixaram o estaleiro ERG1. O modelo também identifica uma quebra estrutural que coincide com a chegada da primeira plataforma ao estaleiro HB, a P53. Conforme pode ser observado na Figura 3, os resultados mostram uma redução permanente de cerca de 100 furtos mensais a partir do início das operações do Polo Naval. 
Figura 3 - Componentes da série de furtos no município de Rio Grande (modelo 2)
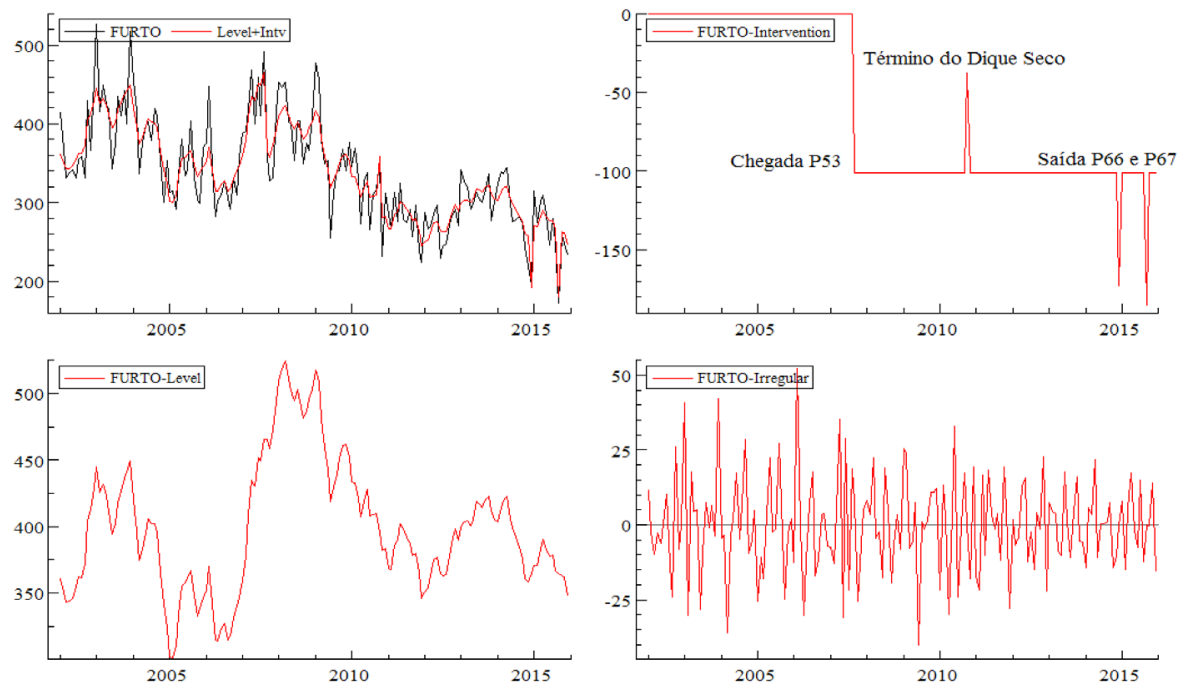

Fonte: Elaboração própria a partir do software Stamp.

Como os resultados indicam mudanças permanentes tanto nos roubos quanto nos furtos registrados no município, que coincidem com eventos relevantes do Polo Naval, cabe discutir a validade interna desses resultados, ou seja, fazer uma avaliação se esses eventos podem ser identificados como a causa para essas mudanças. Em princípio, ao que se sabe, não há nenhum evento paralelo que poderia gerar alterações dessa magnitude nos dois casos. Por exemplo, não há notícia da prisão ou liberdade de alguma quadrilha de criminosos que fosse responsável por uma parcela significativa dos crimes no município. Além disso, os efeitos de choques e de tendência são isolados no modelo estrutural e outras variáveis que poderiam explicar as variações nos crimes contra o patrimônio e que foram omitidas possivelmente tenham um efeito mais lento do que os eventos aqui analisados. Portanto, considerando-se que é sempre recomendável se ter cautela em inferências causais, é possível concluir que os resultados aqui apresentados possuem uma razoável validade interna.

Dando continuidade ao processo de identificação das relações entre a implementação do Polo Naval no município de Rio Grande e os crimes contra o patrimônio, parte-se para a segunda etapa, que consiste em intervenções automáticas. Esse procedimento se justifica porque, apesar de as intervenções manuais terem identificado algumas coincidências importantes, não há garantia de que as mudanças nos crimes ocorram de forma simultânea aos acontecimentos. Nesse caso, é prudente avaliar a existência de quebras estruturais em períodos diferentes como forma de avaliar a robustez dos resultados. Por exemplo, uma possível causa 
para uma transição mais lenta envolve a dinâmica de transição do emprego e da migração em obras desse porte. Havia trabalhadores contratados de várias partes do Brasil e trabalhadores de outros países (chineses e filipinos). Muitos retornavam aos seus locais de origem tão logo a obra fosse concluída, e outros permaneciam no município aguardando uma oportunidade em uma nova construção. Ou seja, a dinâmica das relações entre as condições econômicas e os crimes pode não ser tão direta e imediata e pode passar por uma etapa de transição.

Para se identificar possíveis diferenças e para deixar que os números mostrem o seu comportamento com menos restrições, estimam-se modelos com intervenções automáticas. Esses modelos são superiores em termos de ajustamento, pois seu critério de escolha é puramente estatístico, ou seja, de minimização dos erros do modelo. Os modelos com melhor ajustamento são apresentados na Tabela 3.

Tabela 3 - Resultados das estimações com intervenções automáticas

\begin{tabular}{c|ccccc}
\hline \multicolumn{5}{c}{ Modelo 3 - Roubos } \\
\hline \multirow{2}{*}{$\boldsymbol{T}$} & $\boldsymbol{5}$ & Normalidade \\
& \multirow{2}{*}{168} & $(\mathrm{JB})$ & $\boldsymbol{q}$ & $\boldsymbol{Q}(\boldsymbol{q}, \boldsymbol{q}-\boldsymbol{p})$ & $\boldsymbol{R}_{\boldsymbol{d}}$ \\
& 3 & 1.793 & 24 & 20.555 & 0.52973 \\
& & $(0.4080)$ & & $(0.6648)$ & \\
\hline
\end{tabular}

Efeitos da regressão no estado final do tempo

\begin{tabular}{|c|c|c|c|c|c|}
\hline & Coeficiente & RMSE & Teste $t$ & P-valor & \\
\hline Outlier 2007 (4) & 81.634 & 23.441 & 3.482 & 0.00065 & \\
\hline Outlier 2015 (2) & 163.889 & 23.458 & 6.986 & 0.00000 & \\
\hline $\begin{array}{l}\text { Level Break } \\
2008(11)\end{array}$ & -61.711 & 17.389 & -3.548 & 0.00052 & \\
\hline \multicolumn{6}{|c|}{ Modelo 4 - Furtos } \\
\hline$T$ & $\boldsymbol{p}$ & $\begin{array}{l}\text { (JB) } \\
\text { (JBalida }\end{array}$ & $q$ & $Q(q, q-p)$ & $\boldsymbol{R}^{2}{ }_{d}$ \\
\hline 168 & 5 & $\begin{array}{c}6.170 \\
(0.0457)\end{array}$ & 24 & $\begin{array}{c}34.617 \\
(0.0730)\end{array}$ & 0.24427 \\
\hline \multicolumn{6}{|c|}{ Efeitos da regressão no estado final do tempo } \\
\hline & Coeficiente & RMSE & Teste $t$ & P-valor & \\
\hline Outlier 2006 (2) & 97.827 & 31.253 & 3.130 & 0.00209 & \\
\hline Level Break 2007(9) & -79.978 & 32.552 & -2.457 & 0.01513 & \\
\hline
\end{tabular}

Fonte: Elaboração própria.

Inicialmente, cabe comentar que todos os modelos mostram um bom ajustamento e resíduos normais, ${ }^{6}$ e não autocorrelacionados. Os ajustamentos dos mo-

$6 \quad$ No caso do modelo de furtos, a significância do teste de normalidade de Jarque-Bera é próxima ao limite de $5 \%$ de significância, o que permite inferir que a distribuição dos resíduos é muito 
delos de roubo são superiores aos obtidos pelos modelos de furto, conforme pode ser observado nos coeficientes de ajustamento $\left(R_{d}^{2}\right)$, que comparam o modelo estimado com um passeio aleatório puro. Ambos os modelos mostram um ajustamento melhor do que os modelos com intervenções manuais.

Os modelos com melhor ajustamento para roubos indicam a presença de informações discrepantes em abril de 2007 e fevereiro de 2015, ambas indicam um número de roubos superior aos demais períodos em cerca de 81 e 163 roubos, respectivamente. Para um município que tem uma média mensal inferior a 120 roubos, trata-se de uma alteração relevante. Os modelos também indicam uma possível quebra estrutural que reduz a cerca de 62 roubos mensais no município a partir de novembro de 2008, e nesse período há um forte indício de relação com o Polo Naval, pois esse período é um mês após a saída da P53 e dois períodos após a chegada da P55 no estaleiro HB. Trata-se de uma redução relevante em cerca de $60 \%$ dos roubos que eram observados nos períodos anteriores. Ou seja, mais uma vez os resultados obtidos indicam a existência de um efeito motivação, em que o crescimento do emprego e da renda aumentam os custos de oportunidade de potenciais criminosos e reduzem o crime.

A Figura 4 apresenta graficamente os componentes estimados para o modelo 3 para os roubos registrados no município. Ela permite observar que a série apresenta componentes sazonais regulares (que não alteram seus período e amplitude) com seu ápice nos meses iniciais do ano e com uma redução significativa de roubos nos últimos meses do ano. Além disso, é possível observar no componente de inclinação da série que há uma tendência de aceleração no número de roubos a partir de 2005 até o período em que ocorre a quebra estrutural. A partir desse período, observa-se uma estabilidade na média de roubos nos níveis do início da série, mas com uma posterior aceleração a partir de 2010.

próxima de uma distribuição normal e que o viés gerado pela violação dessa condição é muito pequeno. De qualquer forma, alguma cautela na interpretação dos resultados é sempre recomendável. 
Figura 4 - Componentes da série de roubos no município de Rio Grande (modelo 3)


Fonte: Elaboração própria a partir do software Stamp.

Um aspecto que deve ser considerado é que a velocidade de contratações nesse setor não costuma ser a mesma das demissões. A construção de uma plataforma compreende várias etapas em que, na medida em que vão sendo cumpridas, trabalhadores são dispensados. Ou seja, a contratação de trabalhadores é feita na forma de choque, mas as suas demissões acontecem de forma gradual. Isso talvez explique a aceleração do número de roubos e a sua mudança de nível (intercepto), mas sem que seja possível identificar uma quebra estrutural. Todavia, ao longo do segundo momento de crescimento econômico e do emprego abruptos nos anos de 2012 e 2013, a construção simultânea de três plataformas aparentemente não gerou efeitos no número de roubos.

Ademais, o comportamento do nível da série mostra um crescimento no número de roubos a partir de 2013, quando finda o segundo choque gerado na economia do município pelo Polo Naval. Entre os anos de 2013 e 2014, foram demitidos cerca de 14.500 trabalhadores do setor com a finalização das construções das plataformas P-55, P-58 e P-63 nesse período, segundo dados da RAIS, e as estatísticas desse crime cresceram cerca de 100 roubos mensais. Esse comportamento difere do modelo com intervenções manuais em que foram identificadas quebras estruturais no mesmo período que impulsionaram o número de roubos no município. Todavia, o efeito prático é o mesmo, pois mais uma vez se tem a indicação de um efeito motivação, mas no sentido contrário, ou seja, menos empregos e menos renda associados a mais crimes. Esse resultado é uma evidência semelhante à do estudo de Araújo Júnior e Shikida (2010), pois aparentemente os roubos 
reduziram rapidamente com o aumento da renda e do emprego, mas cresceram de forma gradual quando houve uma redução nessas variáveis. Provavelmente a explicação para esse resultado passa por aspectos que alteram a dinâmica do mercado de trabalho no setor legal, tais como a existência de indenizações por demissões e seguro desemprego.

A Figura 5 mostra os componentes da série de furtos registrados no município com intervenções automáticas. O modelo identifica o mês de fevereiro de 2006 como um mês atípico em que houve cerca de 98 furtos acima do esperado e um componente sazonal que vai perdendo a sua amplitude com o passar do tempo. Da mesma forma que o modelo de roubos, apresenta um número maior de crimes nos meses iniciais e um número menor nos meses finais do ano.

Figura 5 - Componentes da série de furtos no município de Rio Grande (modelo 4)
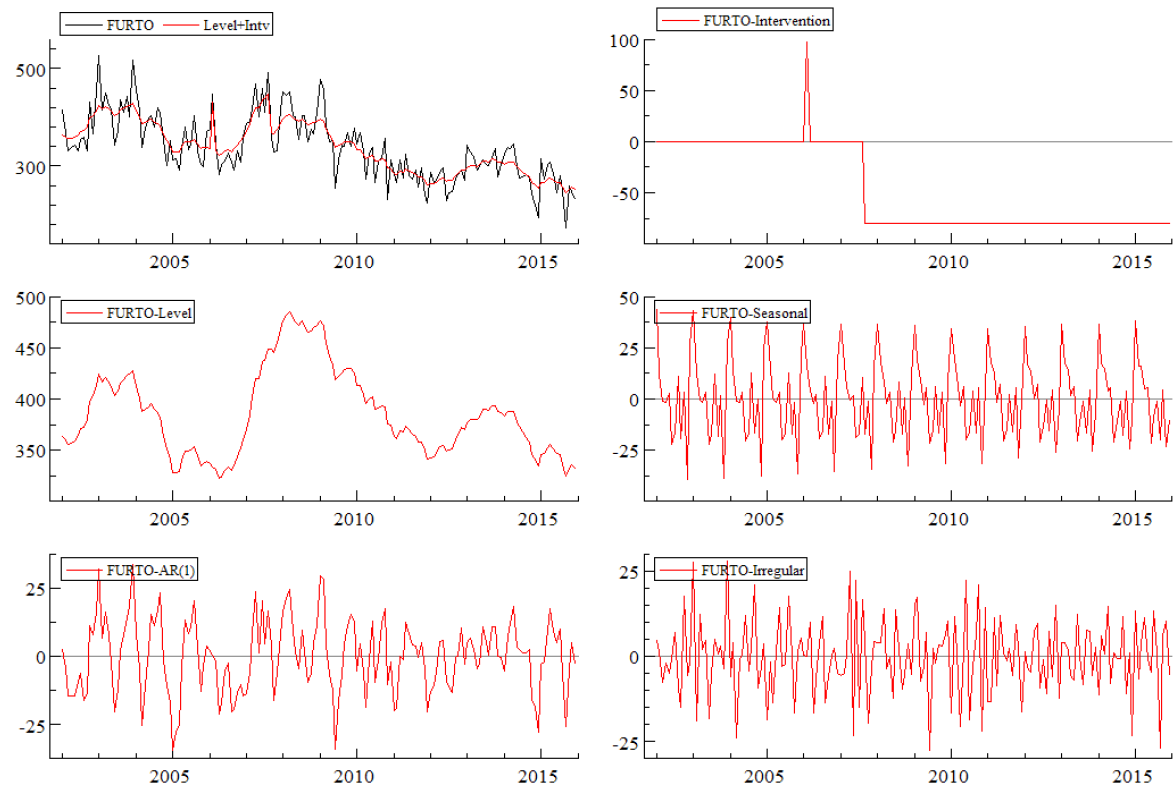

Fonte: Elaboração própria a partir do software Stamp.

No que se refere às quebras estruturais, é possível observar, na Figura 5, que o crime de furto apresenta uma quebra estrutural de nível em setembro de 2007, que reduziu o número de furtos em cerca de 80 furtos mensais de forma permanente. Esse é o mês da chegada da plataforma P53, e tal quebra já havia sido identificada no modelo com intervenções manuais, o que reforça a validade interna desse resultado. Portanto, essa coincidência de datas que aparece nos dois modelos com melhor ajustamento pode ser interpretada como uma relação de causalidade entre as condições econômicas e os furtos registrados no município, uma 
vez que não há qualquer indício de mudanças em outras variáveis que ajudam a explicar os furtos registrados no município que ocorreram de forma simultânea. ${ }^{7}$

Na Figura 5 também é possível observar um componente autorregressivo e um componente de nível que indica uma tendência de decréscimo nos furtos de forma constante a partir de 2008, que corroboram a ideia de associação. $\mathrm{O}$ período em que ocorre a quebra estrutural na série de furtos coincide com o ano de maior crescimento da economia do município, que cresceu cerca de $37 \%$ no ano de 2007. Ademais, essa trajetória de queda a partir desse período culmina no ano de 2012, ou seja, no ano em houve o maior crescimento do emprego formal no município, cerca de $17 \%$. Aparentemente os furtos registrados responderam mais rapidamente às mudanças que ocorreram na economia do município, uma vez que a queda abrupta nos roubos ocorreu mais de um ano após a queda dos furtos, o que pode se tratar de mais uma evidência do efeito motivação no crime de furtos. Um resultado semelhante também foi encontrado, por Arcaro e Oliveira (2016), para os municípios da região metropolitana de Porto Alegre.

\section{Considerações Finais}

A análise da relação entre a criminalidade e variáveis econômicas, tais como renda e emprego, torna-se relevante a partir da ideia de que potenciais criminosos reagem a incentivos e que tais variáveis da conjuntura econômica local podem influenciar suas decisões. O fato de essa relação ser um jogo de forças que ao mesmo tempo podem desincentivar ou incentivar o crime é um forte motivador para que cada vez mais evidências empíricas sejam apresentadas para que se tenha uma compreensão melhor do tema.

Nesse sentido, este estudo teve como objetivo analisar o comportamento dos registros de crimes contra o patrimônio no município de Rio Grande ao longo do tempo e identificar possíveis relações das trajetórias desse tipo de crime com as mudanças econômicas em um ambiente quase experimental gerado pelas mudanças abruptas ocorridas com a implementação do Polo Naval. Para esse fim, utilizaram-se o referencial teórico da abordagem da econômica do crime e a metodologia de decomposição de estruturas de séries de tempo com quebras de estruturais estimadas com uso do FK. Trata-se de um modelo econométrico que poucas vezes é utilizado em estudos de interrupted time series e de Economia do

$7 \quad$ Cabe salientar que problemas causados pela omissão de variáveis relevantes no presente modelo só ocorreriam se tais variáveis omitidas tivessem alguma relação com variáveis exógenas incluídas no modelo, que no presente estudo são dummies que visam captar informações discrepantes (outlier), mudança de inclinação (slope) e mudanças de intercepto (level break). Dessa forma, problemas de endogeneidade por essa causa só ocorreriam se variáveis omitidas tenham sofrido alterações relevantes no exato mês em que ocorreu a mudança no comportamento das séries de crime. 
Crime, mas que é bastante útil considerando-se que os tradicionais modelos Arima utilizam tendências determinísticas para obter as previsões que embasam sua análise contrafactual, enquanto o modelo utilizado no presente estudo é mais flexível e realista ao utilizar previsões baseadas em componentes (inclusive, a tendência) estocásticos. Como a hipótese de exogeneidade estrita do modelo é garantida pela especificação na forma de espaço de estados, uma vez que todos os componentes possuem perturbações independentes dos resíduos do modelo, a única fonte possível de violação dessa hipótese é a existência de alguma variável que tenha uma variação significativa simultânea às mudanças ocorridas na renda, emprego e migração causadas pelo Polo Naval. Essa é a principal vantagem de se trabalhar com um quase experimento, pois se tem uma fonte de variação exógena que permite inferir a respeito de causalidade com menos informações e restrições que outras metodologias.

A principal conclusão e contribuição do estudo é que a implementação do Polo Naval, através de seus impactos na renda, emprego e migração, causaram quebras estruturais identificadas nas séries de crimes contra o patrimônio registrados no município de Rio Grande, uma vez que há a coincidência de datas entre as quebras estruturais identificadas a partir de critérios estatísticos, em especial para o crime de furtos. Todavia, o fato de que a maior parte das intervenções manuais incluídas nos períodos em que houve os principais eventos do Polo Naval não ser significativa estatisticamente, somada ao fato de apenas algumas intervenções obtidas utilizando critérios estatísticos serem significativas em períodos um pouco diferentes das datas dos principais acontecimentos, podem indicar que a dinâmica que envolve as variáveis econômicas e dos crimes pode passar por um período de transição.

Outra conclusão importante é que nos modelos para ambos os crimes, diferentemente da literatura anterior para o estado do Rio Grande do Sul (JOHNSTON; OLIVEIRA, 2016; ARCARO; OLIVEIRA, 2016), foram encontrados evidências de efeito motivação tanto para roubos quanto para furtos, ou seja, uma melhora nas condições econômicas do município com aumento da renda e do emprego foi capaz de reduzir ambos os crimes. Tal resultado, conforme foi destacado no presente estudo, é preponderante na literatura internacional (REILLY; WITT, 1992; GROGGER, 1998; PAPPS; WINKELMANN, 2000; RAPHAEL; WINTER-EBMER, 2001; GOULD; WEINBERG; MUSTARD,, 2002; MACHIN; MEGHIR, 2004; NARAYAN; SMYTH, 2004; MOCAN; BALI, 2010; EDMARK, 2005; DONGIL, 2006) e nacional (PEREIRA; CARREIRA FERNANDEZ, 2000; ARAÚJO JÚNIOR; SHIKIDA, 2010; SANTOS; KASSOUF, 2011). Todavia, os diferentes resultados em estudos realizados no estado do Rio Grande do Sul, em especial para a região metropolitana de Porto Alegre, pode ser uma evidência das diferenças que existem quando ocorrem mudanças graduais e quando ocorrem mudanças abruptas na 
renda, ou seja, mudanças abruptas na renda geram efeito motivação, enquanto mudanças graduais, efeito oportunidade. Entretanto, tal proposição necessita ser melhor estudada, uma vez que também foi identificado um aumento gradual dos roubos tão logo as condições econômicas passaram a não ser mais tão favoráveis.

Por fim, cabe destacar que o trabalho apresenta limitações. Uma delas é que a metodologia utilizada não permite identificar de forma separada os efeitos de mudanças no emprego, renda e migração ocorridos nos períodos estudados. Há a identificação da soma desses efeitos, mas não há garantias de que todos possuam o mesmo sinal (direção). Ademais, a inexistência de informações de municípios comparáveis com a mesma periodicidade do presente estudo não permite testar a possibilidade de que as quebras estruturais identificadas nas séries como efeitos causais do Polo Naval sobre os crimes contra o patrimônio registrados no município tenham sido geradas por outras mudanças exógenas que não foram consideradas no modelo. Embora isso não invalide o processo de identificação, poderia invalidar a relação de causalidade. Todavia, esse tipo de mudança abrupta que gera um ambiente quase experimental é raro e relativamente fácil de ser identificado. Não há conhecimento de que tenha havido alguma mudança nas políticas de segurança pública que possam ter alterado o comportamento dos registros de crimes contra o patrimônio tanto no município de Rio Grande, quanto em municípios comparáveis do estado do Rio Grande do Sul nos períodos em que as quebras estruturais foram identificadas. Mesmo assim, um caminho natural para pesquisas futuras é tentar separar esses efeitos em um modelo que inclua diferentes municípios e variáveis relacionadas ao emprego, renda e migração, mesmo que seja com informações anuais. Dessa forma, será possível ter uma avaliação mais completa de como mudanças estruturais na economia podem alterar os incentivos aos quais os indivíduos estão expostos e, consequentemente, suas decisões a respeito de praticar crimes lucrativos.

\section{Referências}

ARAÚJO JR., A. F.; SHIKIDA, C. D. Recessões e recuperações na atividade econômica: mesmo efeito sobre a criminalidade? Revista Economia 83 Tecnologia, v. 22, p. 47-52, 2010.

ARCARO, D. A.; OLIVEIRA, C. A. Efeitos de dissuasão do mercado de trabalho sobre o crime na região metropolitana de Porto Alegre. Economic Analysis of Law Review, v. 7, n. 2, p. 577-597, 2016.

BECKER, G. S. Crime and punishment: an economic approach. Political Economy, v. 76, p. 169-217, 1968.

BRITT, C. Crime and unemployment among youths in the United States 1958 - 1990: a time series analysis. American Journal of Economics and Sociology, v. 53, p. 99-109, 1994. 
BOX, G. E.; JENKINS, G. M. Time series analysis: forecasting and control. Revised ed. [S.1.]: Holden-Day, 1976.

CAMPBELL, D. T.; ROSS, H. L. The Connecticut crackdown on speeding: time-series data in quasi-experimental analysis. Law and Society Review, v. 3, n. 1, p. 33-54, 1968.

CAMPBELL, D. T.; STANLEY, J. C. Experimental and quasi-experimental designs for research. [S.l.]: Ravenio Books, 2015.

COOK, T. D.; CAMPBELL, D. T. The design and conduct of quasi-experiments and true experiments in field settings. In: DUNNETTE, M. D. (ed.). Handbook of industrial and organizational psychology. Chicago: Rand McNally College, 1976. p. 223-326.

COMMANDEUR, J. J. F.; KOOPMAN, S. J. An introduction to state space time series analysis. Oxford: Oxford University Press, 2007.

CROSBIE, J. Interrupted time-series analysis with brief single-subject data. Journal of Consulting and Clinical Psychology, v. 61, n. 6, p. 966, 1993.

DI TELLA, R.; SCHARGRODSKY, E. Do police reduce crime? Estimates using the allocation of police forces after a terrorist attack. American Economic Review, v. 94, n. 1, p. 115-133, 2004.

DONGIL, K. The effects of economic conditions on crimes. Development and Society, v. 35 , n. 2, p. 241-250, 2006.

DRITSAKIS, N.; GKANAS, A. The effect of socio-economic determinants on crime rates: an empirical research in the case of Greece with cointegration analysis. International Journal of Business and Economic Sciences Applied Research, v. 2, n. 2, p. 51-64, 2009.

DURBIN, J.; KOOPMAN, S. J. Time series analysis by state space methods. 2nd ed. Oxford: Oxford University Press, 2012.

EDMARK, K. Unemployment and crime: is there a connection? Scandinavian Journal of Economics, v. 107, p. 353-373, 2005.

EHRLICH, I. Participation in illegitimate activities: a theoretical and empirical investigation. Journal of Political Economy, v. 81, p. 521-565, 1973.

FUNK, P.; KUGLER, P. Identifying efficient crime combating policies by VAR-estimations: the example of Switzerland. 2000. Mimeo.

GOTTMAN, J. M. Time-series analysis: a comprehensive introduction for social scientists. Cambridge: Cambridge University Press, 1981. v. 400.

GOULD, E. D.; WEINBERG, B. A.; MUSTARD, D. B. Crime rates and local labor market opportunities in the United States: 1979-1997. Review of Economics and Statistics, v. 84, n. 1, p. 45-61, 2002.

GROGGER, J. Market wages and youth crime. Journal of Labor Economics, v. 16, n. 4, p. 756-791, 1998. 
HARVEY, A. C. Forecasting, structural time series models and the Kalman filter. Cambridge: Cambridge University Press, 1989.

HARVEY, A. C.; DURBIN, J. The effects of seat belt legislation on British road casualties: a case study in structural time series modelling. Journal Royal Statistics Society Series A, v. 149, p. 187-227, 1986.

JALLES, J. T. Structural time series models and the Kalman filter: a concise review. Cambridge: University of Cambridge, 2009.

JOHNSTON, F. L.; OLIVEIRA, C. A. Efeitos dissuasórios de ações policiais sobre roubos na Região Metropolitana de Porto Alegre. In: ENCONTRO REGIONAL DA ANPEC SUL, 2016, Florianópolis. [Anais...] Florianópolis: ANPEC, 2016.

KOOPMAN, S. J.; HARVEY, A.; DOORNIK, J.; SHEPHARD, N. Stamp 8: structural time series analyzer, modeller and predictor. [S.1.]: Timberlake Consultants, 2007.

LEVITT, S. D. Alternative strategies for identifying the link between unemployment and crime. Journal of Quantitative Criminology, v. 17, n. 4, p. 377-390, 2001.

LINDEN, A.; ARBOR, A. Conducting interrupted time-series analysis for single-and multiplegroup comparisons. Stata Journal, v. 15, n. 2, p. 480-500, 2015.

MACHIN, S.; MEGHIR, C. Crime and economic incentives. Journal of Human Resources, v. 39, n. 4, p. 958-979, 2004.

MAIA, S. Estatística básica aplicada: movimento de séries de tempo. João Pessoa: Universidade Federal da Paraíba, 2003. Notas de aula.

MELICK, M. D. The relationship between crime and unemployment. The Park Place Economist, v. 11, n. 1, p. 29-36, 2003.

MOCAN, H. N.; BALI, T. G. Asymmetric crime cycles. The Review of Economics and Statistics, v. 92, n. 4, p. 899-911, 2010.

MORETTIN, P. A.; TOLOI, C. M. C. Modelos para previsão de séries temporais. Brasília: CNPq, 1981. vol. 2.

NARAYAN, P. K.; SMYTH, R. Crime rates, male youth unemployment and real income in Australia: evidence from Granger causality tests. Applied Economics, v. 36, p. 2079-2095, 2004.

PAPPS, K.; WINKELMANN, R. Unemployment and crime: new evidence for an old question. New Zealand Economic Papers, v. 34, n. 1, p. 53-71, 2000.

PEREIRA, R.; CARRERA-FERNANDEZ, J. A criminalidade na Região Policial da Grande São Paulo sob a ótica da economia do crime. Revista Econômica do Nordeste, v. 31, n. Especial, p. 898-918, 2000.

RAPHAEL, S.; WINTER-EBMER, R. Identifying the effect of unemployment on crime. Journal of Law and Economics, v. 44, p. 259-283, 2001. 
REILLY, B.; WITT, R. Crime and unemployment in Scotland: an econometric analysis using regional data. Scottish Journal of Political Economy, v. 39, n. 2, p. 213-228, 1992.

SANTOS, M. J.; KASSOUF, A. L. Menos desemprego, maior eficiência da polícia e redução na criminalidade em São Paulo. In: ENCONTRO NACIONAL DE ECONOMIA, 39., 2011, Foz do Iguaçu. [Anais...] Foz do Iguaçu: ANPEC, 2011.

SHADISH, W. R.; COOK, T. D.; CAMPBELL, D. T. Experimental and quasi-experimental designs for generalized causal inference. [S.1.]: Wadsworth Cengage Learning, 2002.

SOOKRAM, S.; MAUKESH, B.; SUMESAR-RAI, K.; SARIDAKIS, G. A time-series analysis of crime in Trinidad and Tobago. [S.1.]: Universidade de West Indies, 2009. Mimeo.

VUJIC, S.; COMMANDEUR, J.J.F.; KOOPMAN, S. J. Intervention time series analysis of crime rates: the case of sentence reform in Virginia, Economic Modelling, v. 57, issue C, p. 311-323, 2016.

ZWIENEN, M. van. Asymmetric crime cycles: an empirical study. 2011. Dissertação (Msc Economcs and Business) - Erasmus University Rotterdam, Rotterdam, 2011.

Recebido em: 18/06/2017. Aceito em: 30/08/2017. 